\title{
An Efficient Disease Detection Technique of Rice Leaf Using AlexNet
}

\author{
Md. Mafiul Hasan Matin, Amina Khatun, Md. Golam Moazzam, Mohammad Shorif Uddin \\ Department of Computer Science and Engineering, Jahanginagar University, Dhaka, Bangladesh \\ Email: mafiulmatinju@gmail.com, amina_bashar@yahoo.com,khokan@juniv.edu, shorifuddin@juniv.edu
}

How to cite this paper: Matin, Md.M.H., Khatun, A., Moazzam, Md.G. and Uddin, M.S. (2020) An Efficient Disease Detection Technique of Rice Leaf Using AlexNet. Journal of Computer and Communications, $\mathbf{8}$, 49-57.

https://doi.org/10.4236/jcc.2020.812005

Received: November 25, 2020

Accepted: December 11, 2020

Published: December 14, 2020

Copyright $\odot 2020$ by author(s) and Scientific Research Publishing Inc. This work is licensed under the Creative Commons Attribution International License (CC BY 4.0).

http://creativecommons.org/licenses/by/4.0/

\begin{abstract}
As nearly half of the people in the world live on rice, so the rice leaf disease detection is very important for our agricultural sector. Many researchers worked on this problem and they achieved different results according to their applied techniques. In this paper, we applied AlexNet technique to detect the three prevalence rice leaf diseases termed as bacterial blight, brown spot as well as leaf smut and got a remarkable outcome rather than the previous works. AlexNet is a special type of classification technique of deep learning. This paper shows more than $99 \%$ accuracy due to adjusting an efficient technique and image augmentation.
\end{abstract}

\section{Keywords}

AlexNet, Leaf Diseases, Disease Prediction, Rice Leaf Disease Dataset, Disease Classification

\section{Introduction}

Bangladesh is a land of agriculture. According to a National Agricultural Census report and the World Bank collection of development indicators in 2020, Bangladesh has 16.5 million farmer families [1] and 37.75 percent population is working in agricultural sector [2]. Rice is the main food harvest of Bangladesh and most probably 75 percent of agricultural land is used for cultivation and we got 28 percent of GDP in 2020 [3]. Rice is found all over the biosphere. It cultivates best in Bangladesh, India, China, Pakistan, Thailand, Burma, Japan, Indonesia etc. There are various diversities of rice in our country. They are principally Aus, Aman, and Boro. There is additional kind of rice called "IRRI" produces well in Bangladesh. We eat rice and get Chira, Khai, Cake, Cloths-paper, Wine etc. from rice. We use its straw as fuel and to make the huts of poor men. Above all, we have to take proper care of its production. But rice leaf diseases decrease 
its production. There are various rice leaf diseases but, in this project work, we have detected three diseases named bacterial blight, brown spot and leaf smut because of the commonness of these diseases in Bangladesh. From [4], Bacterial blight is drawn-out lesions nearby the leaf tips and margins, and chances white to yellow and then grey as a result of fungal attack. Brown spot is a disease of dark brown colored and round to oval shaped scratches on the entire surface of rice leaves. Its scratches are $5-14 \mathrm{~mm}$ long which can cause leaves to wilt. Leaf smut looks like slight black linear scratches on leaf blades, leaf tips may chance grey and dry and these are shown in Figure 1.

To get our expected production, we have to appropriately classify and detect the rice leaf diseases and apply appropriate treatment to abate the losses. Usually, rice disease detection system in our country is done by manual process, then labeling of that diseases by specialists and lastly endorsing proper treatment. These sequences of work are very challenging for massive farms. Besides, it takes huge time and lots of labors. Quite the reverse, capturing the pictures of the invaded zone of the rice leaf and verifying with a learned scheme provides a better way for detection of rice leaf diseases than manual scheme. This paper shows how to correctly detect rice leaf diseases using AlexNet neural network. Not many years ago, we still used small datasets consisting of few numbers of images. These datasets were adequate for machine learning techniques to learn basic recognition tasks. Day by day, life is becoming complex and large problems are come with many more variables. For solving these large problems, we make large datasets like ImageNet and we need an extremely proficient deep learning model like AlexNet [5]. AlexNet is an extremely influential scheme capable of attaining high accuracies on very challenging datasets and it is a foremost architecture for any object-detection task. It has vast applications in the computer vision area of artificial intelligence problems and sooner or later, it may be adopted more than convolutional neural networks for image tasks. For this reasons, AlexNet technique was applied for detecting rice leaf diseases. In AlexNet technique, 70 percent images of our dataset were used as training data and these images were categorized into three object classes such as bacterial blight, brown spot and leaf smut and we got the satisfactory results. The rest of the paper is planned as follows: Segment two contains the literature review. Segment three introduces our proposed work, details of our used dataset and necessary data

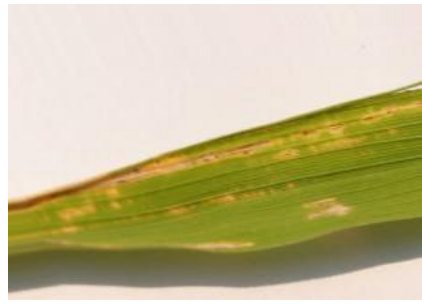

(a)

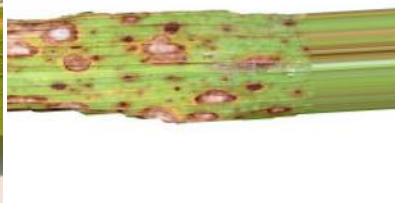

(b)

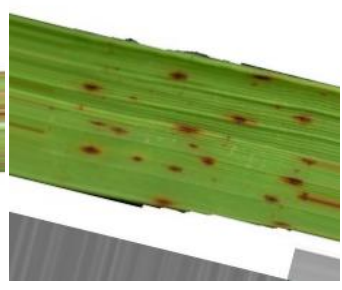

(c)

Figure 1. Three prevalence rice leaf diseases in Bangladesh. (a) Bacterial blight; (b) Brown spot; (c) Leaf smut. 
augmentation. Segment four describes our experimental results and analysis we did and compares our results with other research work. Finally, conclusions and future work are made in Segment five.

\section{Literature Review}

Many researchers worked on this problem and they got different results by applying different methodologies. In paper [6], a model was proposed to categorize the disease based on the expelled RGB percentage value of the invaded zone of rice leaf via image processing techniques and then Naive Bayes classifier was applied to lastly label the diseases into three disease labels named bacterial leaf blight, rice blast and brown spot and this model achieved accuracy of $89 \%$. The authors of [7] worked on random forest, an ensemble learning method to categorize between healthy and diseased leaf and Histogram of Oriented Gradient (HOG) was used for pull out the features of an image and their work obtained 92.33\% accuracy. In [8], the authors proposed a model with 13 diverse categories of diseases of plants using CovNets and they got $96.3 \%$ accuracy. Kurniawati et al. [9] offered a scheme on analyzing the system to distinguish the paddy diseases based on Otsu method and their work has demanded accuracy $87.5 \%$. The mineral shortage identification techniques named K-means clustering and Fuzzy C-means clustering were applied in [10] for rice crop by custom of SVM. At this point, the general identification accuracy by use of SVM with K-means clustering achieved accuracy of $85.05 \%$ and FCM obtained accuracy of $95 \%$. The authors of [11] worked on detection of rice seed disease using SVM and got accuracy of 97.2\%. In paper [12], PCA (Principal Component Analysis) and Neural network were applied for determining rice diseases. This work got accuracy of 95.83\%. H. B. Prajapati et al. [13] used machine learning (ML) and image processing methods for the recognition and classification of rice plant diseases. They applied K-means clustering for image segmentation of rice leaf disease and SVM for classification. They got accuracy of $93.33 \%$ for training data and 73.33\% for testing data. The authors of paper [14] used rice leaf disease detection dataset from the UCI Machine Learning Repository and also used an open source software named WEKA. They applied diverse ML procedures including that of 548 (Decision Tree), Naive Bayes, K-Nearest Neighbour as well as Logistic Regression. They divided the dataset into two parts including that $90 \%$ for training data and $10 \%$ for test data. Among four algorithms, Decision Tree algorithm achieved the highest accuracy of $97 \%$ based on test data. We used same dataset in our proposed work with an efficient technique (AlexNet) and our work shows better accuracy than previous works.

\section{Proposed Work}

This paper shows how to detect rice leaf diseases from a learned AlexNet convolutional neural network to accomplish classification on test data based on training data. The dataset for this task was collected from https://www.kaggle.com 
named rice leaf disease detection [15]. This dataset contained three image files of rice leaf diseases named bacterial leaf blight, brown spot, and leaf smut. Each file had 40 images. From this dataset, we got in total 120 images corresponding to the three rice leaf diseases. These 120 images were too small to work with our proposed technique. For this reason, we increased the size of our dataset by applying image augmentation. After image augmentation, our dataset size was increased to 900 images. In our work, we loaded our custom dataset as an image data-store which mechanically classified the rice leaf diseases based on folder names and stored the data as an image data-store object. The schematic procedures of our proposed work are illustrated in Figure 2. We divided our dataset into two parts as like as training data and test data. $70 \%$ and $30 \%$ data of our dataset were used as training and test data respectively. Therefore, our dataset contained 630 training images and 270 test images.

Finally, AlexNet neural network was applied for prediction of classifying rice leaf diseases. The working procedures of Figure 3 and corresponding actions are shown in Table 1.

This network has five convolutional layers and three fully connected layers. If we remove any of the convolutional layers of our network, then its performance will drastically degrade. The first layer termed as the image input layer used input color images of size 227-by-227-by-3.

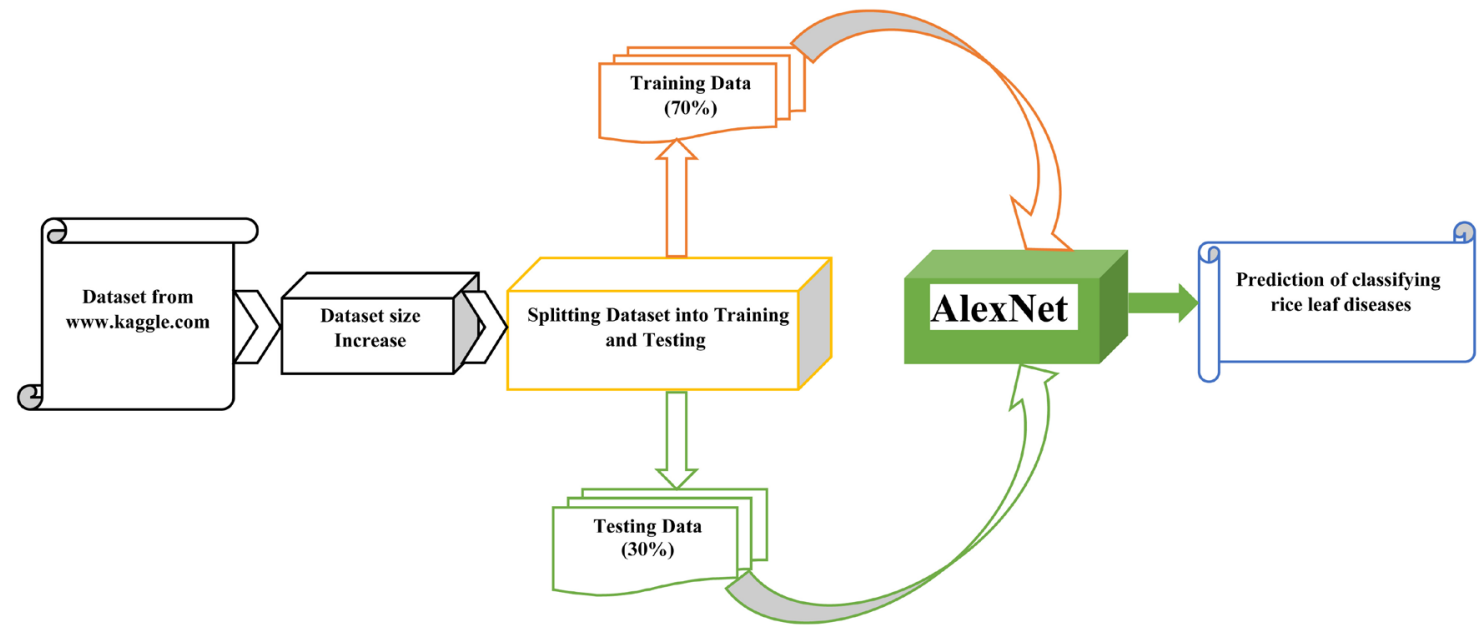

Figure 2. Workflow diagram of our proposed work.

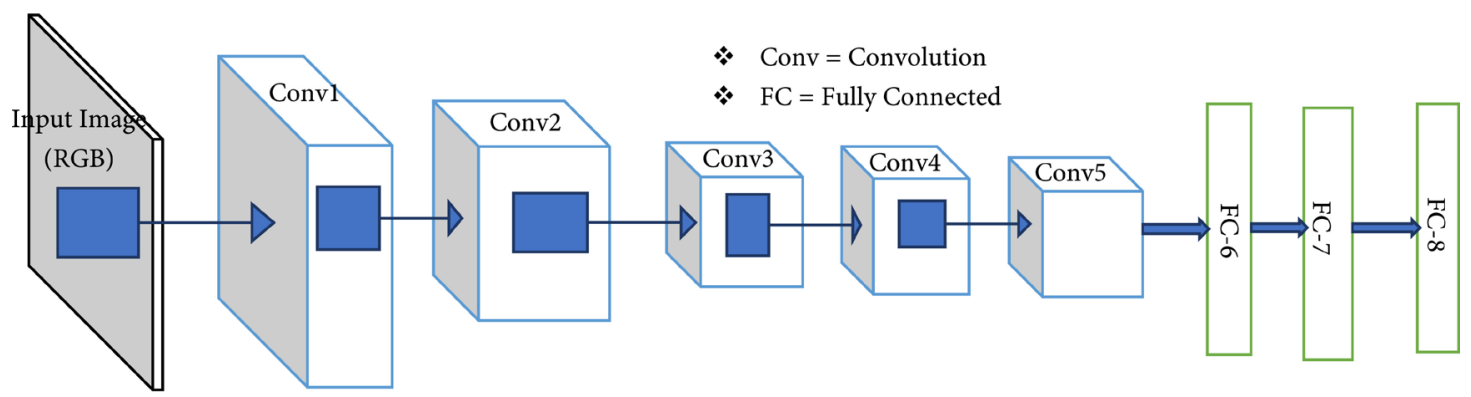

Figure 3. AlexNet neural network architecture. 
Table 1. Layer workflow of our proposed work.

\begin{tabular}{|c|c|c|}
\hline Layer No. & Layer Name & Briefly Explanation \\
\hline 1 & Image Input (data) & $227 \times 227 \times 3$ images with zerocenter normalization \\
\hline 2 & Convolution (conv1) & $9611 \times 11 \times 3$ convolutions with stride $\left[\begin{array}{ll}4 & 4\end{array}\right]$ and padding $\left[\begin{array}{llll}0 & 0 & 0 & 0\end{array}\right]$ \\
\hline 3 & ReLU (relu1) & ReLU \\
\hline 4 & Cross Channel Normalization (norm1) & cross channel normalization with 5 channels per element \\
\hline 5 & Max Pooling (pool1) & $3 \times 3$ max pooling with stride $\left[\begin{array}{ll}2 & 2\end{array}\right]$ and padding $\left[\begin{array}{llll}0 & 0 & 0 & 0\end{array}\right]$ \\
\hline 6 & Convolution (conv2) & $2565 \times 5 \times 48$ convolutions with stride $\left[\begin{array}{ll}1 & 1\end{array}\right]$ and padding [ 2 \\
\hline 7 & ReLU (relu2) & ReLU \\
\hline 8 & Cross ChannelNormalization (norm2) & cross channel normalization with 5 channels per element \\
\hline 9 & Max Pooling (pool2) & $3 \times 3$ max pooling with stride $\left[\begin{array}{ll}2 & 2\end{array}\right]$ and padding $\left[\begin{array}{llll}0 & 0 & 0 & 0\end{array}\right]$ \\
\hline 10 & Convolution (conv3) & $3843 \times 3 \times 256$ convolutions with stride $\left[\begin{array}{ll}1 & 1\end{array}\right]$ and padding $\left[\begin{array}{ll}1 & 1\end{array}\right.$ \\
\hline 11 & ReLU (relu3) & ReLU \\
\hline 12 & Convolution (conv4) & $3843 \times 3 \times 192$ convolutions with stride $\left[\begin{array}{ll}1 & 1\end{array}\right]$ and padding $[1$ \\
\hline 13 & ReLU (relu4) & $\operatorname{ReLU}$ \\
\hline 14 & Convolution (conv5) & $2563 \times 3 \times 192$ convolutions with stride $\left[\begin{array}{ll}1 & 1\end{array}\right]$ and padding $[1$ \\
\hline 15 & ReLU (relu5) & ReLU \\
\hline 16 & Max Pooling (pool5) & $3 \times 3$ max pooling with stride $\left[\begin{array}{ll}2 & 2\end{array}\right]$ and padding $\left[\begin{array}{llll}0 & 0 & 0 & 0\end{array}\right]$ \\
\hline 17 & Fully Connected (fc6) & 4096 fully connected layer \\
\hline 18 & ReLU (relu6) & ReLU \\
\hline 19 & Dropout (drop6) & $50 \%$ dropout \\
\hline 20 & Fully Connected (fc7) & 4096 fully connected layer \\
\hline 21 & ReLU (relu7) & ReLU \\
\hline 22 & Dropout (drop7) & Dropout \\
\hline 23 & Fully Connected (fc8) & 1000 fully connected layer \\
\hline 24 & Softmax (prob) & Softmax \\
\hline 25 & Classification Output (output) & crossentropyex with tench and 999 other classes \\
\hline
\end{tabular}

The last three layers of Figure 3 were constructed for 3 classes. These three layers were adjusted for the rice leaf disease detection and extracted all layers except the last three from our AlexNet neural network. In our work, classification and detection tasks were transferred by changing the last three layers with a fully connected layer, a softmax layer, and a classification output layer. The options of the new fully connected layer were itemized along with the new data. Finally, our model successfully classified the three commonness rice leaf diseases and predicted the detection accuracy. 20 sample images of three rice leaf diseases are displayed in Figure 4.

\section{Results and Discussions}

Figure 5 shows the overall training and testing progress of Figure 3. We set initial 


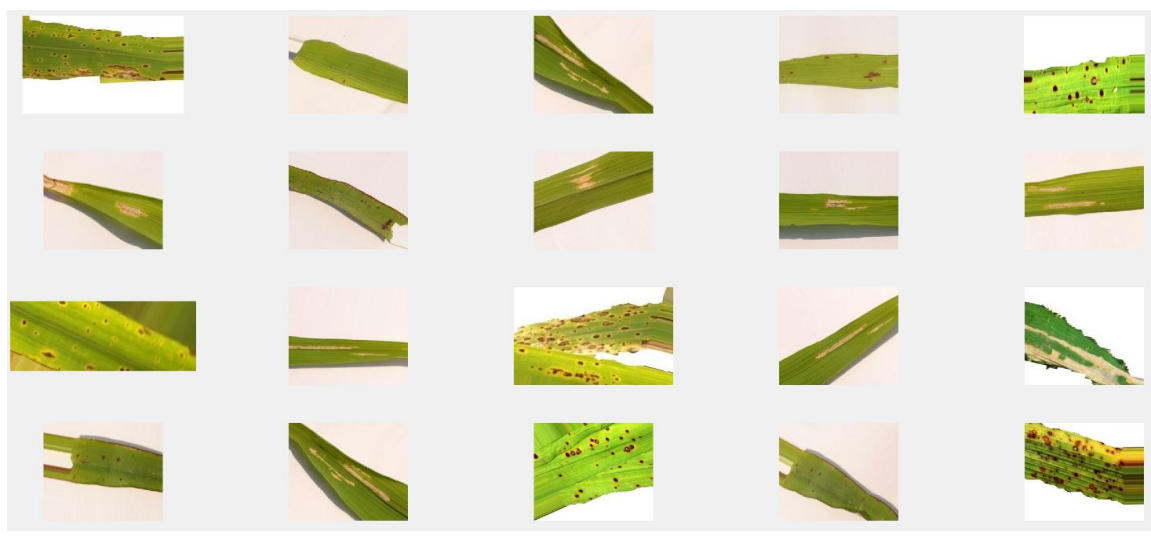

Figure 4. Sample images of our dataset.

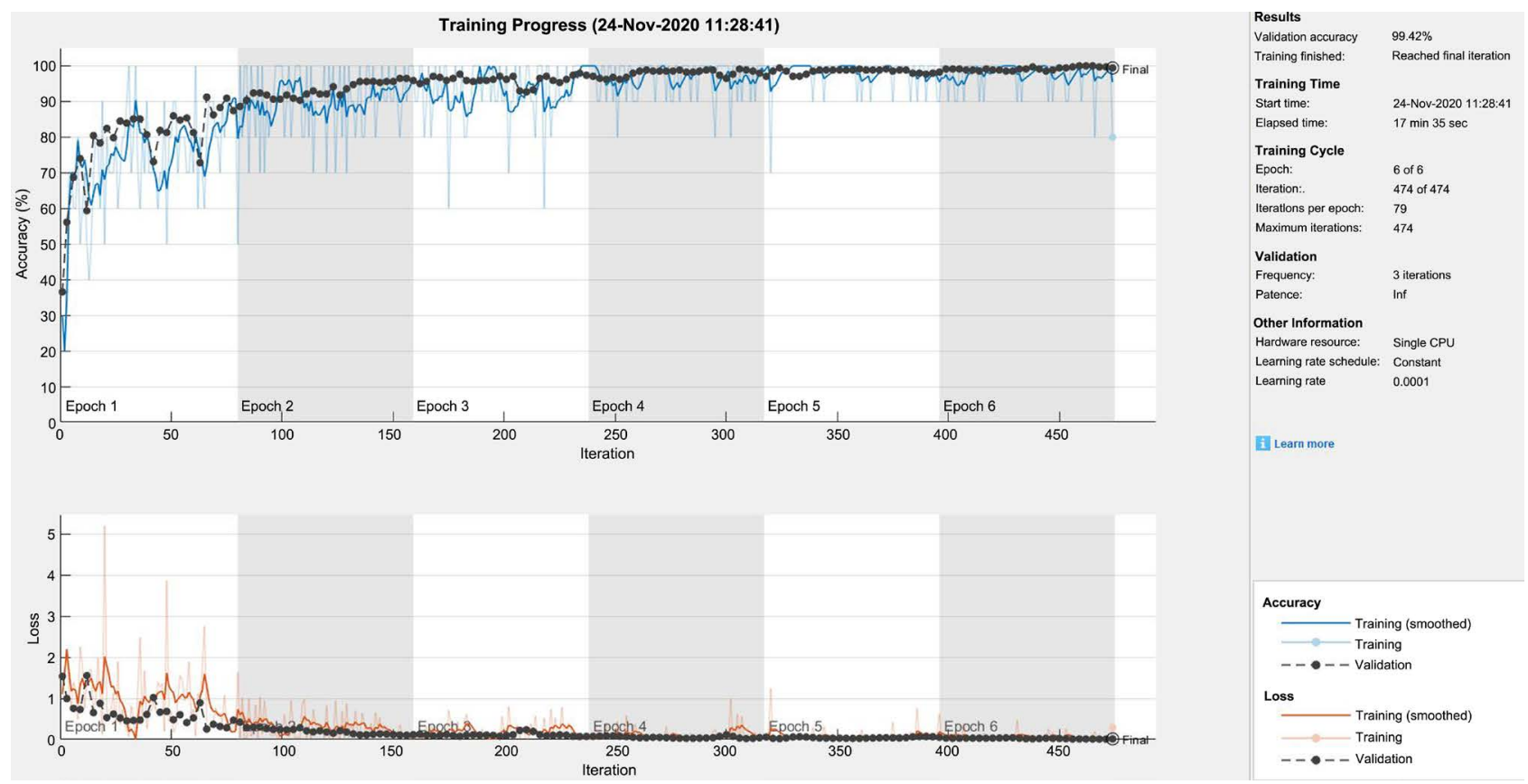

Figure 5. Training progress and results of our proposed work.

learning rate 0.0001 for steady learning in the transferred layers. We set epoch of size 6 as a full training cycle on our entire training dataset and the validation frequency was every 3 iterations during training data. In our work, the elapsed time 17 minutes 35 seconds because of hardware constraint. The hardware constraint means our model by default uses a GPU (Graphics Processing Unit) because our dataset contained colored images with high resolution. But our device had no GPU. For unavailable of GPU, our model used CPU (Central Processing Unit) and took more than few times to execute our entire task. This elapsed time may vary from devices to devices. The execution environments are illustrated in Figure 5.

This training progress demonstrates that we classified the validation images using the adjusted AlexNet technique. The graph of Figure 5 shows the three rice leaf diseases detection accuracy and loss compared to corresponding itera- 
tion. In first epoch, our model was trained by small number of data, that's why the accuracy based on test data was not good and had too much loss. But when gradually increased the number of epochs, the accuracy also increased due to enlarging trained data and decreased the validation loss. Six trial validation images with their predicted labels are exhibited in Figure 6. Our proposed technique detected the three rice leaf diseases with their corresponding levels.

We compared the validation accuracy of our work among previous works. From the Figure 7, we can see that our accuracy is the highest accuracy among all the research papers enlisted in our reference section.

From the graph of Figure 7, we can also see that paper [8] [11] [12] and [14] achieved the satisfactory results with different approaches. After studied their works, we also tried to work with the same problem domain using different approach named AlexNet and we expected high accuracy. Finally, we got $99.42 \%$ accuracy of rice leaf disease detection that our network predicted appropriately.

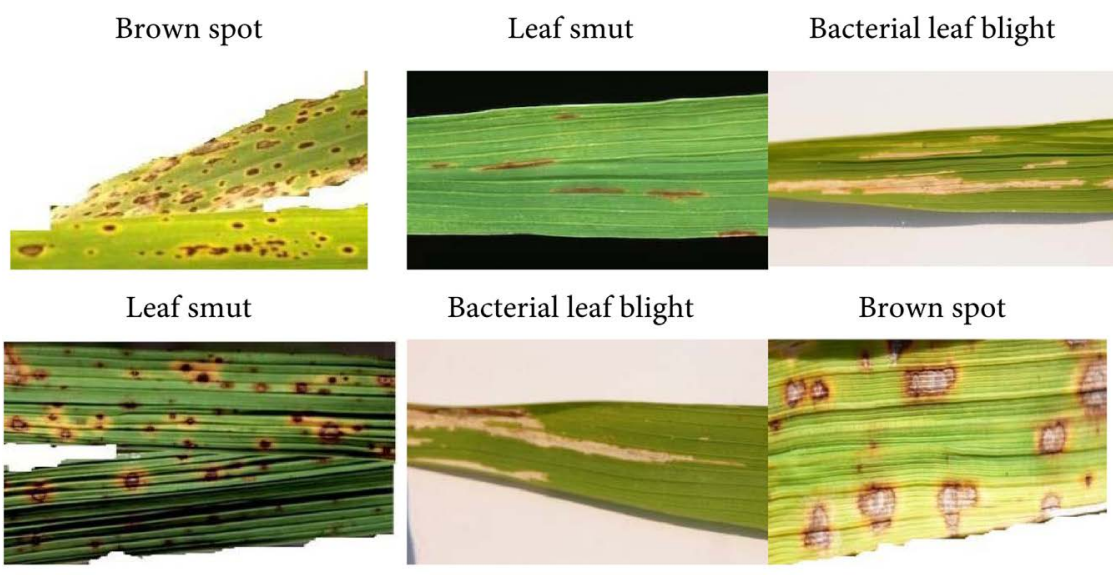

Figure 6. Three rice leaf disease detection.

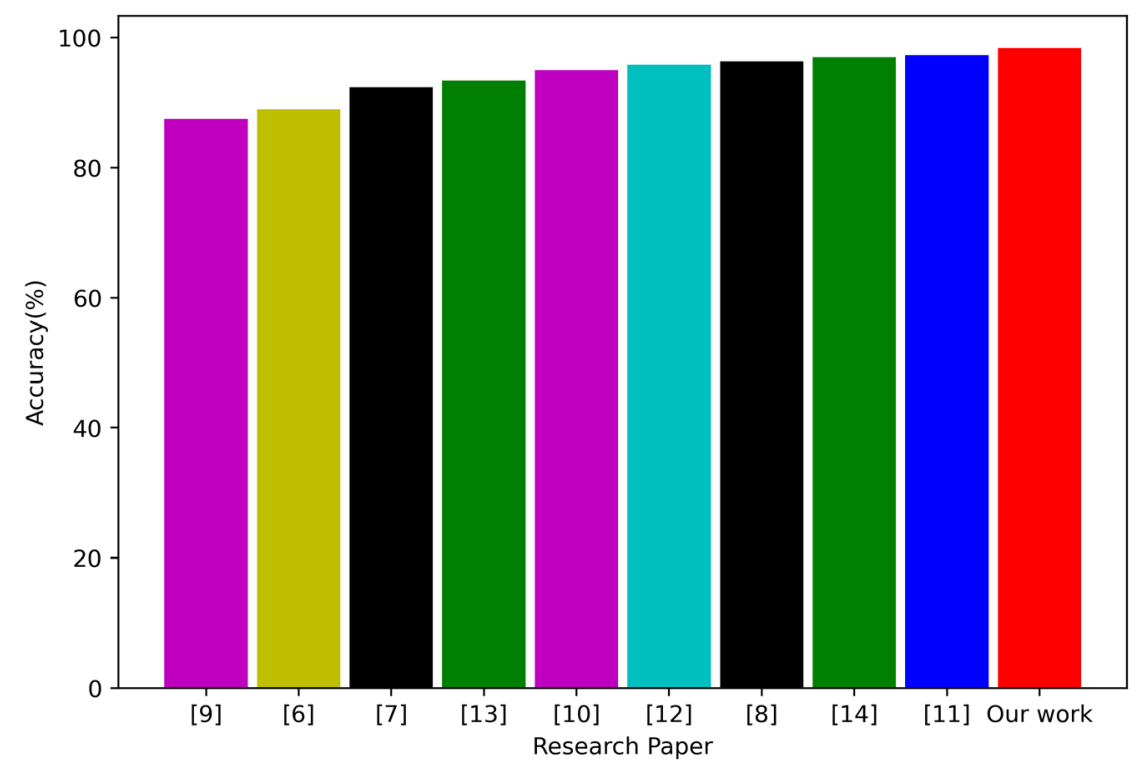

Figure 7. Accuracy comparison graph among research papers and our proposed work. 


\section{Conclusion and Future Work}

In this paper, we detected the three prevalence rice leaf diseases using AlexNet neural network and our result provides improved accuracy compared to previous works of applied different approaches. In our experiment, AlexNet was adopted more than CNNs for image tasks. This paper has weighty economic significance for not only Bangladesh but also other rice production-based countries. In future, we still have an opportunity of using the K-means clustering algorithm, Fuzzy c-mean clustering, CART etc. with fuzzy inference system (FIS) for judgment in context of accuracy and process time.

\section{Conflicts of Interest}

The authors declare no conflicts of interest regarding the publication of this paper.

\section{References}

[1] Dakha Tribunal. https://www.dhakatribune.com/bangladesh/agriculture/2019/10/27/agricultural-cen sus-report-16-5-million-farmer-families-in-bangladesh

[2] Trading Economics. https://radingeconomics.com/bangladesh/employment-in-agriculture-percent-of-t otal-employment-wb-data.html

[3] Wikipedia. https://en.wikipedia.org/wiki/Rice production in Bangla

[4] Rice Disease Identification Photo Link. http://www.agri971.yolasite.com/resources/RICE\%20DISEASE\%20IDENTIFICATI ON.pdf

[5] Krizhevsky, A., Sutskever, I. and Hinton, G.E. (2017) ImageNet Classification with Deep Convolutional Neural Networks. Communications of the ACM, 60. https://papers.nips.cc/paper/2012/file/c399862d3b9d6b76c8436e924a68c45b-Paper. pdf

[6] Islam, T., Sah, M., Baral, S. and Choudhury, R.R. (2018) A Faster Technique on Rice Disease Detection Using Image Processing of Affected Area in Agro-Field. 2018 Second International Conference on Inventive Communication and Computational Technologies (ICICCT), Coimbatore, 20-21 April 2018, 62-66. https://doi.org/10.1109/ICICCT.2018.8473322

[7] Maniyath, S.R., Vinod, P., Niveditha, M., Pooja, R., Shashank, N., Hebbar, R., et al., (2018) Plant Disease Detection Using Machine Learning. 2018 International Conference on Design Innovations for 3 Cs Compute Communicate Control (ICDIBC), Bangalore, 25-28 April 2018, 41-45.

[8] Sladojevic, S., Arsenovic, M., Anderla, A., Culibrk, D. and Stefanovic, D. (2016) Deep Neural Networks Based Recognition of Plant Diseases by Leaf Image Classification. Computational Intelligence and Neuroscience, 2016, Article ID: 3289801. https://doi.org/10.1155/2016/3289801

[9] Kurniawati, N.N., Abdullah, S.N.H.S., Abdullah, S. and Abdullah, S. (2009) Texture Analysis for Diagnosing Paddy Disease. 2009 International Conference on Electrical Engineering and Informatics, Selangor, 5-7 August 2009, 23-27. https://doi.org/10.1109/ICEEI.2009.5254824 
[10] Sethy, P.K., Negi, B. and Bhoi, N. (2017) Detection of Healthy and Defected Diseased Leaf of Rice Crop using K-Means Clustering Technique. International Journal of Computer Applications, 157, 24-27. https://doi.org/10.5120/ijca2017912601

[11] Liu, Z.Y., Shi, J.J., Zhang, L.W. and Huang, J.F. (2010) Discrimination of Rice Panicles by Hyperspectral Reflectance Data Based on Principal Component Analysis and Support Vector Classification. Journal of Zhejiang University Science B, 11, 71-78. https://doi.org/10.1631/jzus.B0900193

[12] Xiao, M., Ma, Y., Feng, Z., Deng, Z., Hou, S., Shu, L. and Lu, Z. (2018) Rice Blast Recognition Based on Principal Component Analysis and Neural Network. Computers and Electronics in Agriculture, 154, 482-490.

https://doi.org/10.1016/j.compag.2018.08.028

[13] Prajapati, H.B., Shah, J.P. and Dabhi, V.K. (2017) Detection and Classification of Rice Plant Diseases. Intelligent Decision Technologies, 11, 357-373.

https://doi.org/10.3233/IDT-170301

[14] Ahmed, K., Shahidi, T.R., Irfanul Alam, S.M. and Momen, S. (2019) Rice Leaf Disease Detection Using Machine Learning Techniques. 2019 International Conference on Sustainable Technologies for Industry 4.0 (STI), Dhaka, 24-25 December, 1-5. https://doi.org/10.1109/STI47673.2019.9068096

[15] Rice Leaf Diseases Data Set. https://www.kaggle.com/vbookshelf/rice-leaf-diseases 